\title{
La moda contemporánea en Brasil: para escapar del Siglo XX
}

\section{Avelar, Suzana}

Resumen:

Este artículo presenta una lectura posible del consumo de moda en la cultura digital. Además de una discusión sobre las redes, aquí presentamos algunas relaciones de creación y consumo atravesadas por la cultura digital, centradas en la construcción del cuerpo vestido por algunos actores de la industria de la moda y fuera de ella. Por lo tanto, el artículo se circunscribe al concepto de moda, señalando algunas discusiones sobre el consumo y las subjetividades, culminando en dos aspectos de las líneas de fuga en el contexto de la industria de la moda.

Palabras clave: moda -

Cuadernos del Centro de Estudios de Diseño y Comunicación Nº76

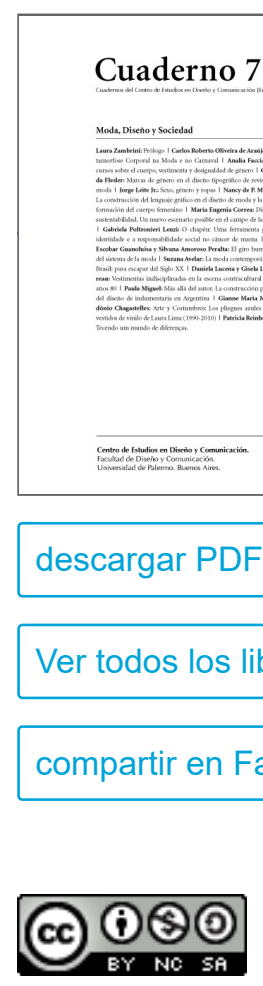

Esta obra está bajo una Licencia Creative Commons Atribución-NoComercialCompartirlgual 4.0 Internacional

contemporánea - cultura - digital.


Semiótica en la PUC, São Paulo. Curadora del proyecto "A mão na moda" del museo CASA. Autora del libro Moda, globalização e novas tecnologias por la editorial Estação das Letras e Cores. Profesora en la Universidade de São Paulo en los cursos de grado y postgrado en Textil y Moda.

Introducción

La idea que la moda es algo que tiene como punto de partida las vanidades, que se caracteriza por los valores más superficiales y triviales, siempre ha estado presente a lo largo del siglo XX y todavía resuena en el momento actual. Lo que podemos ver es que, de acuerdo con el documental The True Cost1, la industria del consumo de vestuario es la segunda más grande en el mundo, solo superada por la industria petrolera. En este artículo, abordaremos un concepto particular de la moda, con el fin atravesar sus dinámicas y prestar atención a los aspectos sociales presentes en sus prácticas. 
A continuación, puntualizaremos algunas breves cuestiones relevantes en la construcción del cuerpo vestido, atravesadas por las prácticas de la confección. Esto significa considerar la ropa que viste al cuerpo en sus modos de producción por manos humanas, en sus afectos y las existencias en ella implicada. Muchos grupos que participan en la confección de la ropa, hoy, han estado trabajando de manera de resaltar el elemento humano de esta práctica, produciendo modos que pueden contribuir materialmente en la producción de lo que se convirtió en nuestro ser social y cultural: la ropa en el cuerpo vestido. Por último, indicaremos algunas personas y colectivos que han estado activos dentro de la moda y de su industria, nombrando a las manos implicadas y sus contextos sociales.

\section{Concepto de moda}

Para los historiadores de la moda François Boucher (1996) y François Baudot (2008), aún es posible discutirse la moda, aunque su emergencia sea atribuida al Renacimiento, junto con el concepto de "individuo" que piensa más allá de los dogmas religiosos. Tal discusión debe entrar en el territorio de los conceptos filosóficos y sociológicos. Sin embargo, por la forma como la moda y su industria se han construido en la sociedad moderna, permeada por la práctica del consumo y la cultura de la imagen, su comprensión parece estar estructurada de ese modo.

Para este artículo, esperamos contribuir a la comprensión sobre moda en la manera cómo nos relacionamos a través del cuerpo vestido en determinados espacios y contextos de nuestras construcciones corporales y lo que eso conlleva. Los espacios urbanos y digitales son aquellos donde suceden encuentros frecuentes y de distintas naturalezas, favoreciendo lo que Gabriel Tarde ha llamado "espíritu de la moda" -la imitación más allá de los modelos familiares- inevitablemente provocando contaminaciones e intensidades diversas (formas breves, violentas y contradictorias) no lineales.

El concepto mismo de moda se encuentra ahí, en lo que se hace colectivo, se disemina y afecta a otros de manera similar. Sin la imitación parece imposible el evento moda. El deambular por la ciudad, como ha sugerido Gehl (2014), ya que la ciudad está construida por las personas, puede ser para ellas la posibilidad de permear modos heterogéneos de circulación, que operan en el espacio y son por él atravesados. También debemos considerar los paseos digitales: aunque unidimensionales, hiper-textuales, pueden ser una publicación de uno mismo, siendo la ropa un elemento de primordial importancia.

Nunca se ha ejercido tanto la moda como ahora con los software de grandes volúmenes de datos (Big Data), ya que "moda" es la media de referencia entre el más allá y el más acá, que ahora es tomada por la aplicación de los grandes volúmenes de datos en la composición del cuerpo vestido - nuestro ser cultural.

Comprender la moda de esa manera parece un poco más contundente, especialmente para hablar de la práctica del vestirse en lo contemporáneo, adentrando o no a la industria de la moda. Los estudios sobre la moda deben realizarse en la interdisciplinariedad, en la cooperación entre las distintas áreas2, como ocurrió en la creación del traje de baño Speedo, que ha sido una colaboración entre el diseñador y los científicos3 .

Aquí tratamos la representación visual en pos de cómo esperamos ser vistos, el cuerpo y aquello que lo involucra. Es decir, se coloca en un lugar central en estos estudios, y pueden ser "leídos" a través del encuentro 
la sociología, las teorías de la cultura, la psicología, los modos de producción y las relaciones colocadas en la práctica del consumo, así como a través de los espacios en donde estos cuerpos circulan, entre otros.

Algunos puntos sobre la moda y la cultura digital

La moda impregna diversos segmentos culturales en la práctica de la constitución de nuestro ser social y cultural4, protagonizando la relación de sí al otro, en una tensión entre la individuación y la colectivización, en la que actúa como elemento constitutivo del sujeto. Para Gilda de Mello e Souza (1993) y Gabriel Tarde, la moda puede ser comprendida como la imitación que se intensifica en las zonas urbanas, más allá del núcleo familiar. En la actualidad, se puede considerar esta observación válida todavía, señalando que los modos de imitación y poder ocupan otros lugares, a través de diferentes discursos y herramientas, más allá de aquel disciplinar panóptico. En la sociedad del control, las formas de poder pueden ocurrir de maneras más sutiles y permeables, flexibles 5 , anónimas y dispersas 6 , tornando dóciles los cuerpos y sus representaciones de diversas maneras y en constante mutación.

Nadav Rochman (2014), analizando la práctica de la selfie en un proyecto dirigido por Lev Manovich, nos muestra que tal exposición puede funcionar como un intento de agrupar afinidades a través grandes volúmenes de datos en una composición de la imagen fotográ- fica de sí. Indica Peter Pál Pelbart que esta práctica de monitoreo permanente nos invade cada día convirtiéndose en parte de nuestra existencia.

En el caso de la moda, destacamos la posibilidad de nuevas experiencias visuales, mientras que se multiplican exponencialmente normas iguales. En el primer caso, tales experiencias pueden alterar los caminos de la autorepresentación, dando lugar a nuevas formas de resistencia, escapando de la reproducción de lo mismo. Entendemos aquí "lo mismo" como aquello que viene disfrazado de discurso del cambio como carácter innovador, y que con el tiempo reproduce a sí mismo sin ninguna nueva conexión; la repetición del vacío disfrazada de novedad.

Breve consideración sobre blogs de moda

Un hecho significativo de hoy puede ser la proliferación de los blogs de moda. Al principio, los blogs sirvieron como maneras de subvertir la industria de la moda que, desde la década de 1960, se encontraba establecida a partir de la dinámica muy bien concatenada entre los talleres de estilo, las industrias químicas, textiles y de confección, las revistas femeninas y el consumo. Podemos decir que la relación de consumo de moda estaba basada en el aprendizaje de un siglo, en el que el consumidor delegó a los creadores el poder de decidir los modelos de novedades para la construcción de su cuerpo público, que circulaba en la escena de la ciudad. Esto significa decir que parece haber existido un contrato, informal y efectivo, entre el individuo y la industria de la moda, que servía a una sociedad burguesa y después de clase media, de masa, en la que el valor de la imitación actuó de manera significativa en las relaciones de clase y en los esquemas culturales. Recordando a Foucault (1981), difícilmente se construye una relación de poder sin el consentimiento de todas las partes, aunque esto ocurra de diferentes maneras, complejas y coercitivas.

Los blogs de moda casi rompieron con esta lógica sedimentada en el momento en que se valen de la autonomía o del "do it yourself" proporcionados por la redes digitales. Estos blogs utilizaron las brechas para crear espacios de la construcción de sí, fortaleciendo la confusa separación entre lo público y lo privado. El poder disciplinario 
de esta industria parece haberse rendido a la potencia que la cultura digital favorecía. Sin embargo, las personas clave de la industria de la moda subrepticiamente capturaron muchos protagonistas de los blogs, y los han atraído para su seductora acción de engendrar modelos sociales. Muchos blogs se valen de productos de las marcas mundialmente legitimadas, con el discurso de los "nuevos looks", y parecen actuar solamente como un elemento fortalecedor del consumo esquizofrénico de las mismas cosas, para a menudo disfrazar el egocentrismo narcisista de sus protagonistas.

No fue este el caso del blog "Un año sin Zara", diseñado por Joanna7 . Ella propuso pasar un año sin comprar ropas, recombinando y cambiando los significados de lo que ya estaba allí, subvirtiendo el consumo desenfrenado de ropas y adornos corporales, en el corazón de la práctica del fast fashion8 . Lo que queda evidente aquí es la capacidad de reinvención de uno mismo a través las brechas del consumo rápido, fácil y modelado; la calidad de la creación del sujeto sugiere actuar mayormente fuera de los manuales para, de manera contradictoria, su "éxito personal" (signifique lo signifique eso en un manual...).

Acerca de las brechas

Una advertencia sobre el comercio debe ser hecha: no presentamos aquí un discurso irreductible contra el consumo de la moda. El consumo puede ser entendido como una práctica en la que se concretan relaciones sociales de variadas naturalezas.

En su texto sobre el comercio, Heliana Comin Vargas (2012) nos cuenta que en el origen del comercio está el estímulo a la coexistencia de culturas diversas, pues el comercio ocurre en locales donde hay cruces de rutas y, por lo tanto, espacios de relaciones sociales. A pesar de las grandes desigualdades sociales existentes en razón del capital, es posible encontrar en el consumo tipos de enlaces con el otro, que promueven tipos de relaciones más colectivas y menos individualistas, miradas más cuidadosas sobre otras realidades. Ese es el propósito de este texto: presentar la moda como una práctica más amplia del cuerpo social y un campo sujeto a la creación de líneas de fuga.

Es evidente que hemos vivido un consumo exagerado sin mucha reflexión acerca de las condiciones humanas en la producción y en el consumo mismo, superando, muchas veces, los límites tolerables o aceptables de todos los que están involucrados en la fabricación de las ropas que visten el cuerpo.

Un hecho que parece sorprendente es que con la competencia de los productos chinos (y de muchos otros en todo el mundo), muchos países han señalado que tal vez la única manera de competir frente al abuso es empezar a tener en cuenta el trabajo humano. Eso parece haber disparado la difusión de la defensa de principios más razonables en los modos de producción en la industria de la moda, teniendo en cuenta la calidad de las condiciones de trabajo.

Como sugiere Axel Hornneth (2003), el individuo se torna consciente de su subjetividad porque él, bajo la presión de un problema práctico a ser resuelto, se ve obligado a rediseñar creativamente sus interpretaciones de la situación.

De ese modo, para bien y para mal, se resiste al consumo de ropa éticamente incorrecto, pero encuentra todavía una manera de seguir proveyendo moda en el mismo sistema lógico de esta industria, creando un nuevo 
valor para la ropa, a saber, la producción de ropa ubicada en países que primero valorizaron su mano de obra y que eran protagonistas de los discursos de tendencias: un monopolio de toda la cadena textil.

Mucho de eso puede y debe ser discutido, pero nos gustaría seguir con la presentación de acciones más sensibles en el plan de la moda hecha en Brasil, teniendo en cuenta características y contextos de las personas involucradas en el proceso entero. En el presente escenario, visto desde la perspectiva de la cultura digital, destacamos la inmediatez siempre inminente en las relaciones sociales; se espera que las intenciones se transformen en acciones efectivas y regulatorias de un modelo medio de las relaciones humanas en sus diversas prácticas.

Lo que importa aquí es como la conciencia del valor del otro, la consideración por el ser humano de una manera razonable, empieza a ser percibido en el contexto de la moda.

Nuestras prácticas cotidianas son atravesadas por el consumo desde los tiempos en que el comercio protagonizaba encuentros entre culturas y pueblos diversos 9 . Hoy, la compra de mercancía tiene sus caracterizaciones simbólicas y espectaculares10 permeadas por las esquizofrenias humanas que siempre se localizan en algún lugar del sujeto, disparando dinámicas y problemas propios de la situación del consumo11.

Aun así, ubicamos en la moda prácticas de consumo que tienen en cuenta el factor humano involucrado en la creación y producción de aquello que compondrá el cuerpo que se hace culturalmente visible.

Pensar el proyecto de colección, proceso creativo, hoy, es pensar al mismo tiempo modos de producción respetuosos con la mano de obra empleada. En este momento hay grupos dentro de la propia industria de la moda que colocan los abusos de derechos humanos involucrados en la producción de ropas, como se puede ver en el documental The True Cost (Andrew Morgan, 2015) y en el trabajo de la organización Fashion Revolution12.

Los casos presentados a continuación nos muestran diferentes maneras de construir adornos para el cuerpo, en los que el trabajo humano surge como parte de la constitución de su significado: Fernanda Yamamoto y Flavia Aranha, diseñadoras de moda, constituyen sus colecciones revelando la mano de obra como parte del proceso creativo, utilizando la moda como una herramienta para el reconocimiento de personas, a través del enlace entre la mano de obra, la humanidad, el afecto, en la reconstrucción del ideal de "producto". Pensar el proceso creativo y productivo de manera interdependiente significa remodelar el concepto de "producto" como referencia simbólica.

Yamamoto se aproxima a ciertas comunidades de artesanas con la finalidad de presentar su idea y verificar si les gustaría fabricar el encaje necesario para la colección, como es el caso de su colección de invierno 2016. Además de hacer visitas regulares a las comunidades de Cariri, en el estado de Paraiba (región nordeste de Brasil), Fernanda Yamamoto toma decisiones juntamente con las modistas y fabricantes de encaje, y difunde este proceso junto con la formalización de la colección en desfile en Sao Paulo Fashion Week. Invitar a estas mujeres para desfilar algunas de las piezas fue una manera más de resaltar que el trabajo es realizado por personas con know-how que participa en la generación de ingresos para muchas familias en el país. 
Flavia Aranha, diseñadora de moda formada por la Facultad Santa Marcelina, entiende que la moda no es solamente el propio producto que viste el cuerpo en sus representaciones simbólicas y colectivas; para ella la moda está constituida por el elemento humano que la produce no sólo en el proceso creativo. Flavia Aranha encuentra otro camino para incluir el valor humano como parte de la constitución de la idea del producto. Ella también trabaja con comunidades de tejedoras en sus ciudades o pueblos, y opta por promover su colección no con desfiles pero a través la producción de catálogos, que presentan las piezas y las personas involucradas en el proceso.

Este también es el rol de la organización Fashion Revolution13 en favor del reconocimiento de la mano de obra involucrada en la industria del vestuario, buscando tornar evidente ese valor humano, y que puede ser citado junto al de otra organización similar, la Fair Trade14. Dos personas que también deben ser incluidas en este artículo son Renato Imbroisi y Silvia Sasaoka, quienes trabajan en la mejora de los productos de las comunidades artesanas; sus intervenciones se realizan de manera participativa, es decir, son llevadas a cabo en conjunto con los individuos de la comunidad en cuestión.

La actuación de Marcia Aguiar, psicóloga y coordinadora de la organización no gubernamental Croph15, y de Francisca Dantas Mendes, investigadora y profesora de la Universidad de Sao Paulo, es notable en lo que se refiere a un trabajo que contribuye para la búsqueda de nuevas destinaciones para los residuos textiles producidos principalmente en el barrio de Bom Retiro, en Sao Paulo. Los residuos textiles se utilizan en el taller de alfombras y en objetos hechos con cañamazo. Este trabajo tiene al menos dos aspectos que deben ser mencionados, a saber: un destino para el resto de tejido dispensado por la industria de confección; y un trabajo de producción de objetos ejecutado por personas que viven en situación de calle en el amparo Sao Francisco.

La obra de la artista Mónica Nador y del Jardim Miriam Arte Clube16, que comprende la producción estética combinada con una producción social, sugiere el arte como construcción social del sujeto, el sujeto individual constituyente y constituido por un sujeto colectivo.

La marca de zapatos Ciao Mao, de Priscila Callegari, dispone lado a lado la creación y la fabricación del producto en el mismo ambiente, diseñándolo al mismo tiempo que compone sus formas, volúmenes, materiales y colores. Por otra parte, los materiales elegidos deben ser aquellos que provienen de residuos textiles y del cuero que no utiliza Cromo VI, elemento muy contaminante en el entorno de las curtidurías.

Por último, vale mencionar el proyecto del Museo A Casa, realizado en el año 2000, cuyo proyecto "Moda y Artesanía"17 tenía como idea central volver a inserir la artesanía del encaje de bolillos en el mercado. El trabajo fue realizado con la Asociación Artesanas del Encaje de Morro da Mariana, en el estado brasileño de Piauí (región nordeste de Brasil), por Walter Rodrigues, diseñador de moda. La comunidad de las artesanas se encuentra en la ciudad costera de Paraiba, con gran parte de su generación de ingresos derivada de los encajes de bolillos y de la pesca. A través del nombre legitimado del diseñador de moda, de la exposición y del documental, la asociación se hizo conocida como polo de encaje de bolillos en Brasil. Hubo trabajo colectivo y mucho intercambio de conocimiento relacionado al mercado, así como las técnicas que aseguraron la independencia de la asociación en relación a los otros participantes del proyecto. La primera idea el proyecto siempre fue trabajar el significado del encaje de bolillos para que las artesanas tomasen decisiones y supieran lo que estaba ocurriendo en cada etapa. Dieciséis años más tarde, la asociación continúa siendo gestionada por las propias artesanas y eligiendo los trabajos que van a ser hechos por ellas. 
En las prácticas descritas anteriormente, vimos proyectos que aproximan diseñador y comunidades de manera más participativa, teniendo en cuenta la realidad diaria de las personas que participan de todo el proceso, desde el diseño y hasta la fabricación de las piezas de vestuario. En el próximo abordaje, vamos a mostrar ejemplos de acciones también contemporáneas que hacen uso de la lógica de red de comunicación digital con el fin de potencializar los encuentros de ideas y acciones, de manera colectiva. Lo que queremos abordar aquí es el probable comienzo, o intensificación, del desmembramiento de la "estética el héroe", en la que la realización en la moda está centrada en un único "genio", en la figura de un "salvador", dotado de poder institucional.

Las ferias colectivas, realizados cooperativamente, parecen ser una de las maneras de difundir las diversas manos de obra participantes en la construcción de un objeto de consumo. Percibimos aquí la potencialidad de las redes sociales y otros medios digitales en favor de la promoción y del estímulo de hacerse públicos todos los implicados. Otro aspecto a complementar, es que estas ferias promueven las conversaciones en presencia física, que también pueden continuarse por los medios digitales. En este caso, se compone una proximidad física y digital, tornando más tangible el encuentro de las manos del proceso creativo y la realización de objetos y colecciones de moda.

La difusión y la generación de ideas pueden cruzarse de manera más inmediata a través de las plataformas colectivas, las aplicaciones, encuentros de variada naturaleza, aprovechando otros dispositivos con respecto a la fabulación de la creatividad. Parece entonces que el acto creador se revela en miradas conjuntas, con particularidades subjetivas que asumen la práctica colectiva, sin la necesidad de apoyarse en la lógica egotista del genio creativo, exitoso en los lugares legitimados de la industria de la moda. Este modo de entender la creación de moda puede proveer diversos caminos que engendran nuevos formatos que están fuera de una concepción tradicional de la industria de la moda, desplazando poderes centralizados para nuevas (u otras) maneras de fabricarse la moda.

Ejemplos de estas brechas pueden ser representados por el espacio del Armario Cápsula18 y el Mercado de Pulgas19. El primero ofrece un fortalecimiento de la disminución del consumo de piezas de moda mediante el intercambio de ideas colectivas con un guardarropa reducido. El segundo también promueve una disminución del consumo, pero a través de un evento anunciado por la red social Facebook, realizado en lugares de la ciudad de Sao Paulo, que estimula el intercambio y la venta de piezas de ropa entre los participantes. Los pequeños productores también se pueden encontrar en los bazares organizados por la red, en general, por varias personas.

\section{Conclusiones}

El concepto de la moda presentado al principio de este artículo se afirma más y más, una vez que la moda- es decir, la forma como nos relacionamos a través del cuerpo vestidodesacopla la industria jerárquica y se manifiesta independientemente de los lugares formales. Obviamente, eventos así han ocurrido a lo largo de la historia, como las boutiques de diseñadores - por ejemplo, Biba, Mary Quant, Vivienne Westwood (en los años 1960 y 1970 en el Reino Unido), Cooperativa de Moda (en los años 1980), Mercado Mundo Mix y Escola de Divinos (en la década de 1990), todos en Sao Paulo, entre otros. Estos pueden ser considerados como líneas de fuga en un contexto de moda centrada en un modus operandi muy bien organizado entre las industrias textiles y 
de confección, al lado de las revistas femeninas y de los talleres de estilo, así como la patente francesa de haute couture.

Vemos, por lo tanto, en los dos momentos referenciados aquí (en el inconformismo con el uso de mano de obra de manera inescrupulosa y en las formas colectivas de creación), que existen otras formas de acción para el cuerpo vestido, ya enunciadas y que se revelan desmembradas del rígido monopolio secular de una industria de la moda.

Notas

1. Andrew Morgan, 2015.

2. Santos, M. (2004). Por uma geografia nova. São Paulo: EDUSP. 4a ed.

3. https://www.swimmingworldmagazine.com/news/industry-news-speedo-unveilsteam-usa-Izr-racer/, visitado el 11-04-2016.

4. Ash, J. E Elizabeth Wilson (1993). Chic thrills. London: Collins.

5. Deleuze, G. (1992). Post-scriptum sobre a sociedade de controle. In: Conversações 1972-1990. Rio de Janeiro: Ed 34. Trad. Peter Pál Pelbart.

6. Pelbart, P. P. (2015). Biopolítica. In: Revista da Sala Preta do PPGAC da USP. Vol. 15 No 2.

7. http://uasz.com.br/, visitado el 12-04-2016.

8. Machado, W. S. (2013). Do podrinho ao vintage: a visualidade dos blogs de moda e a publicidade em imagens de look do dia. Dissertação de mestrado. FAV/ UFG. https://cultura visual.fav.ufg.br/p/6212-dissertacoes

9. Vargas, H. C. (2012). Comércio e cidade. In: http://www.memoriasdocomerciosp. museudapessoa.net/public/Arquivos/COM\%C3\%89RCIO\%20E\%20CIDADE\%20 UMA\%20RELA\%C3\%87\%C3\%830\%20DE\%200RIGEM.pdf

10. Debord, G. (1997). A sociedade do espetáculo. Rio de Janeiro: Contraponto.

11. Pelbart, P. P. (2015). Biopolítica. In: Revista da Sala Preta do PPGAC da USP. Vol. 15 No 2.

12. http://fashionrevolution.org/, visitado el 20-03-2016.

13. http://fashionrevolution.org/country/brazil/, visitado el 12-04-2016.

14. http://www.fairtrade.net/, visitado el 12-04-2016.

15. http://www2.croph.org.br/, visitado el 12-04-2016.

16. http://jamac.org.br/, visitado el 12-04-2016. 
17. Suzana Avelar actuó en este proyecto como curadora. http://www.acasa.org.br/evento. php?id=43, visitado el 12-04-2016.

18. https://www.facebook.com/groups/armariocapsula/, visitado el 08-04-2016.

19. https://www.facebook.com/events/1678023809134659/, visitado el 08-04-2016.

Bibliografia

Avelar, S. (2011). Moda, globalização e novas tecnologias. São Paulo: Estação das Letras e Cores. 2a ed.

Boucher, F. (1996). A history of costume in the West. London: Thames and Hudson.

Hornneth, A. (2003). Luta por reconhecimento. São Paulo: Editora 34.

Rainho, M. Do C. T. (2010). "Notas sobre moda, juventude e paradigmas teóricos”. In: Revista Ciência e Cultura, vol. 62, No 2. Disponible en: http://cienciaecultura.bvs.br/ scielo.php?script=sci_arttext\&pid=S0009-

67252010000200013

Rochman, N. (2014). Imagined data communities. Disponible en: http://d25rsf93iwlmgu. cloudfront.net/downloads/Nadav_Hochman_selfiecity.pdf

Souza, Gilda de M. e (1993). O espírito das roupas. São Paulo: Companhia das Letras.

Vargas, H. C. (2012). "Comércio e cidade". IN: Memórias do comércio paulista: Guia do acervo. Disponible en: http://www.museudapessoa.net/public/editor/mioloprova_-_mdcomercio_-_guia.pdf , visitado el 28/01/2016.

Um ano sem Zara, http://uasz.com.br/, 28/01/2016.

Dechanelnalaje, https://dechanelnalaje.wordpress.com/, 28/01/2016.

Abstract: This paper presents a possible reading on the consumption of fashion in the digital culture. Besides a discussion about networks, it approaches some relations between creative process and consumption, which are crossed by the digital culture, focused on the construction of the dressed body by some actors within the fashion industry and outside it. The paper circumscribes the concept of fashion and highlights some debates on consumption and subjectivity, and finally indicates two aspects of the lines of flights in the context of the fashion industry.

Key words: fashion - contemporary - culture - digital.

Resumo: Este artigo apresenta uma possível leitura para o consumo de moda na cultura digital. Para além de uma abordagem sobre as redes, apresentamos aqui algumas relações de criação e consumo atravessadas pela cultura digital, centrada na construção do corpo vestido através de alguns atores da indústria da moda e fora dela. Para tanto, o artigo circunscreve o conceito de moda, apontando algumas discussões acerca do consumo e das subjetividades, culminando em dois aspectos de linhas de fuga no contexto da indústria da moda. 
Palavras chave: moda - contemporânea - cultura - digital.

[Las traducciones de los abstracts fueron supervisadas por el autor de cada artículo]

La moda contemporánea en Brasil: para escapar del Siglo XX fue publicado de la página 133 a página141 en Cuadernos del Centro de Estudios de Diseño y Comunicación №76 